\title{
Investigation of the Young's modulus and thermal expansion of amorphous titania-doped tantala films
}

\author{
Matthew R. Abernathy, ${ }^{1,2, \star}$ James Hough, ${ }^{2}$ lain W. Martin, ${ }^{2}$ Sheila Rowan, ${ }^{2}$ \\ Michelle Oyen, ${ }^{3}$ Courtney Linn, ${ }^{4}$ and James E. Faller ${ }^{5}$ \\ 'LIGO, Caltech, 1200 E. California Blvd., Pasadena, California 91125, USA \\ ${ }^{2}$ SUPA, University of Glasgow, University Ave., Glasgow G12 8QQ, UK \\ ${ }^{3}$ Cambridge University, Engineering Department, Trumpington Street, Cambridge CB2 1PZ, UK \\ ${ }^{4}$ Embry-Riddle Aeronautical University, 3700 Willow Creek Road, Prescott, Arizona 86301, USA \\ 5 JILA, University of Colorado, 440 UCB, Boulder, Colorado 80309, USA \\ ${ }^{*}$ Corresponding author: abernathy_m@ligo.caltech.edu
}

Received 16 January 2014; accepted 2 April 2014;

posted 17 April 2014 (Doc. ID 204591); published 14 May 2014

\begin{abstract}
The current generation of advanced gravitational wave detectors utilize titania-doped tantala/silica multilayer stacks for their mirror coatings. The properties of the low-refractive-index silica are well known; however, in the absence of detailed direct measurements, the material parameters of Young's modulus and coefficient of thermal expansion (CTE) of the high refractive index material, titania-doped tantala, have been assumed to be equal to values measured for pure tantala coatings. In order to ascertain the true values necessary for thermal noise calculations, we have undertaken measurements of Young's modulus and CTE through the use of nanoindentation and thermal-bending measurements. The measurements were designed to assess the effects of titania-doping concentration and postdeposition heat-treatment on the measured values in order to evaluate the possibility of optimizing material parameters to further improve thermal noise in the detector. Young's modulus measurements on pure tantala and $25 \%$ and $55 \%$ titania-doped tantala show a wide range of values, from 132 to $177 \mathrm{GPa}$, which are dependent on both titania concentration and heat-treatment. Measurements of CTE give values of $(3.9 \pm 0.1) \times 10^{-6} \mathrm{~K}^{-1}$ and $(4.9 \pm 0.3) \times 10^{-6} \mathrm{~K}^{-1}$ for $25 \%$ and $55 \%$ titania-doped tantala, respectively, without dependence on post-deposition heat-treatment. (c) 2014 Optical Society of America OCIS codes: (310.6870) Thin films, other properties; (310.3840) Materials and process characterization; (310.1860) Deposition and fabrication; (160.2750) Glass and other amorphous materials.

http://dx.doi.org/10.1364/AO.53.003196
\end{abstract}

\section{Introduction}

The current generation of interferometric gravitational wave detectors, including the Advanced LIGO [1] and Advanced Virgo [2] detectors, are undergoing construction and are expected to reach design sensitivity in the next few years. An important limiting noise source in the detectors is the thermal noise

$1559-128 \mathrm{X} / 14 / 153196-07 \$ 15.00 / 0$

(C) 2014 Optical Society of America arising from the coatings used to make the mirrored test masses reflective at a wavelength of $1064 \mathrm{~nm}$. These high-reflectivity coatings are made from alternating layers of a high index of refraction-ion-beam sputtered (IBS) amorphous titania-doped tantala ( $\mathrm{Ti}: \mathrm{Ta}_{2} \mathrm{O}_{5}$ ) and low index IBS amorphous silica $\left(\mathrm{SiO}_{2}\right)$, with the layer structure optimized to reduce thermal noise while maintaining the requisite reflectivity [3]

In order to calculate the thermal noise that arises in the interferometers a priori, the thermomechanical properties of the coating materials need to be well 
known. In order to calculate the thermo-optic noise [4] of the coatings, knowledge of the heat capacity, CTE, thermo-optic coefficient, thermal conductivity, Young's modulus, and Poisson ratio of the coating material is required. In order to calculate the Brownian thermal noise [5], the Young's modulus, Poisson ratio, and mechanical loss of the coating material are also needed. Furthermore, calculation of the mechanical loss from various "ringdown" measurement techniques [6,7] requires knowledge of the coating material's Young's modulus and Poisson ratio.

While the properties of silica are fairly well known, the properties of tantala, and especially titania-doped tantala, have rarely been measured. In some cases, there has even been controversy regarding some measurements. In the case of the CTE, measurements have ranged from $-4.4 \times 10^{-5} \mathrm{~K}^{-1}$ for ion-assisted e-beam-sputtered tantala [8], to $2.4 \times 10^{-6} \mathrm{~K}^{-1}$ for IBS tantala [9]. Measurements made by Braginsky and Samoilenk $\bar{k}$ [10] suggested that the CTE of tantala was roughly $\overline{(5} \pm 1) \times 10^{-6} \mathrm{~K}^{-1}$. This new measurement was used to support the use of a value of $3.6 \times$ $10^{-6} \mathrm{~K}^{-1}$ within the LIGO community for IBS pure tantala, and it has since been commonly used [4,11, and others]. However, this value was calculated [9] from measurements of the temperature coefficients [12] and normalized thermo-optic coefficients [13] and was not a direct measurement of thermal expansion, nor were these values measured on IBS coatings.

For the Young's modulus of tantala, the value of $140 \mathrm{GPa}$ is most often used in analysis of coating mechanical loss and estimates of coating thermal noise. The article by Martin et al. [14] is often cited $[6,7,15,16$, and others]; however, this paper only displays plots of indentation modulus as a function of indentation depth using a microindentation system, and these plots have not been fully analyzed to give an appropriate coating of Young's modulus. Other measurements support the value of $140 \mathrm{GPa}$ using nanoindentation, including measurements of $140 \pm$ $15 \mathrm{GPa}$ [17] and $143 \mathrm{GPa}$ [18]. Unfortunately, these measurements do not offer a complete analysis of the substrate effects on the nanoindentation measurements.

The purpose of the measurements made in this paper is twofold: first, to remove any further controversy regarding the Young's modulus and CTE of IBS tantala films; and second, to measure these properties for the case of the IBS titania-doped tantala coatings used in advanced detectors. As dopant level and post-deposition heat treatment have been identified as variables that can affect the Brownian thermal noise in the detectors $[7,19,20]$, coatings with a range of dopant levels and heat-treatments were also studied here.

\section{Measurement}

All coatings were produced by CSIRO (Commonwealth Scientific and Industrial Research Organization) and deposited upon both 1 in. silica discs and the silicon cantilevers commonly used in mechanical- loss measurements [7]. The pure tantala samples were prepared as part of one coating run and were heat-treated to $300^{\circ} \mathrm{C}, 400^{\circ} \mathrm{C}, 600^{\circ} \mathrm{C}$, and $800^{\circ} \mathrm{C}$. It was previously shown that the $800^{\circ} \mathrm{C}$ sample had begun to crystallize [20]. The titania-doped samples were made during a separate coating run and were either left untreated or heat-treated to $300^{\circ} \mathrm{C}, 400^{\circ} \mathrm{C}$, or $600^{\circ} \mathrm{C}$. The untreated samples are often referred to as as-deposited (AD); the deposition temperature was $100^{\circ} \mathrm{C}$, so for the purpose of this paper, the results are considered as heat-treated to this temperature. Additional heat-treatment was carried out by annealing samples in air for $24 \mathrm{~h}$. All of the titaniadoped tantala samples were found to be amorphous. Titania-doped samples were either $25 \%$ or $55 \%$ titania, as measured by metal cation. All coatings were measured using ellipsometry to be $\sim 500 \mathrm{~nm}$ thick.

\section{A. Nanoindentation}

Nanoindentation is a technique developed to measure the mechanical properties of small volumes of materials in a simple fashion [21]. These properties are measured by making indentations at the nanometer scale and recording the load, $P$, and displacement, $h$, response, as the indenter is driven into and withdrawn from the material. An example of the nanoindentation load-displacement curves taken into tantala can be seen in Fig. 1. In this example, the indents are made using the standard load-hold-unload cycle. A load is applied to the indentation tip, forcing it into the sample and increasing the displacement. During the loading phase, work is done as the sample is both elastically and plastically deformed. During the hold phase, the force is held constant, but the sample continues to deform due to creep effects, which arise due to the movement of the material within the specimen under high pressure. During the unloading phase, the load is reduced, and the indentation tip is withdrawn from the sample. This phase is characterized by having only an elastic response. We therefore analyze the unloading phase in order to measure the Young's modulus of the material.

Using this technique, indentation measurements were made using the Hysitron TI-700 Ubi, located at the University of Cambridge, with a diamond Berkovich pyramidal tip. Effective moduli, composed

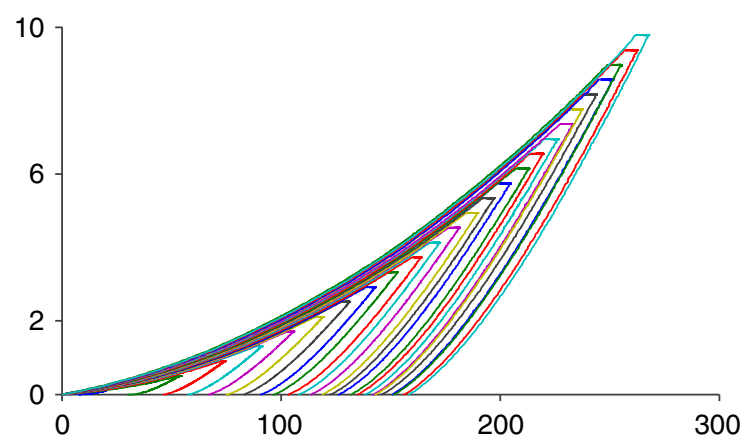

Fig. 1. Example of the load-displacement curves used in calculating the Young's modulus at one position on a film of pure tantala deposited on a silica substrate and heat-treated to $600^{\circ} \mathrm{C}$. 
of the coating and substrate Young's moduli, were extracted from the indentation data using the method of Oliver and Pharr [22]. Once the load-displacement data is recorded, the elastic modulus is determined from

$$
E^{*}=\frac{\sqrt{\pi}}{2} \frac{d P}{d h} \frac{1}{\sqrt{A}}
$$

where $A$ is the projected area of contact under load, $d P / d h$ is the slope of the load-displacement curve at the beginning of the unloading phase, and $E^{*}$ is the combined modulus of the sample and indenter:

$$
\frac{1}{E^{*}}=\frac{1-\nu_{i}^{2}}{E_{i}}+\frac{1-\nu_{s}^{2}}{E_{s}}
$$

Here $E$ is the Young's modulus, and $\nu$ is the Poisson's ratio of the sample and indenter, marked with subscripts $s$ and $i$, respectively.

The value of $d P / d h$ is generally extracted from the data by fitting an empirically derived equation,

$$
P=\alpha\left(h-h_{f}\right)^{m},
$$

to the unloading portion of the curve. Here $\alpha$ and $m$ are the fitting constants, and $h_{f}$ is the displacement at zero load on the unloading curve. Once fit, the derivative of the $\mathrm{P}-\mathrm{h}$ relation is taken at the maximum value of $\mathrm{h}, h_{\max }$, to give the value of $d P / d h$.

The area function is calculated by making a series of indents into a fused quartz reference sample with a known hardness and Young's modulus, solving Eq. (1) for $A$, and fitting the following equation [23]:

$$
A\left(h_{c}\right)=C_{1} h_{c}^{2}+C_{2} h_{c}+C_{2} h_{c}^{1 / 2}+C_{4} h_{c}^{1 / 4}+\ldots,
$$

where $h_{c}$ is the elastic contact depth from Ref. [22], and the values of $C_{n}$ are adjusted by the fit. In general, fits are performed only up to $C_{4}$.

For a thin coating on much thicker substrate, if the Young's moduli of the coating and substrate differ, the modulus measured using the Oliver and Pharr method will vary with indentation depth [24]. This is due to the increasing influence of the substrate as the load is increased. In order to minimize this influence, it is often suggested that indents be made such that $h_{\max }$ is less than $10 \%$ of the thickness of the coating, $t_{c}$ [23]. While this is generally acceptable for coatings greater than about a micron, it is not practical on thinner coatings where the errors in the area function and surface defects begin to have an effect at very small indentation depths [25].

The coating modulus was extracted from the values taken from the Oliver and Pharr method using the model of Song and Pharr [26,27]. In the Song and Pharr model, the elastic moduli of the film and substrate are added in series and weighted by a factor dependent upon the indentation area:

$$
\frac{1}{E^{\prime}}=\frac{1}{E_{s}}+\left(\frac{1}{E_{c}}-\frac{1}{E_{s}}\right) I_{0}(t / a) .
$$

Here the subscripts $c$ and $s$ represent the coating and substrate, respectively, and $I_{0}(t / a)$ is a weighing function that is equal to 1 for shallow indents and 0 for deep indents and is given by the equation [28]:

$$
\begin{aligned}
I_{0}(t / a)= & \frac{2}{\pi} \arctan (t / a)+\frac{1}{2 \pi(1-\nu)} \\
& \times\left[(1-2 \nu)(t / a) \ln \left(\frac{1+(t / a)^{2}}{(t / a)^{2}}\right)-\frac{t / a}{1+(t / a)^{2}}\right],
\end{aligned}
$$

where $a$ is the radius of a circle with the equivalent area as the projected area of indent, $\pi a^{2}=A\left(h_{c}\right)$, and $t$ is the difference between the thickness of the coating and the contact depth of the indent [27]. A plot of $E^{\prime-1}$ against $I_{0}$ for a number of indents made at different depths will yield a linear relationship with the y-intercept of $E_{s}^{-1}$ and a value of $E_{c}^{-1}$ at $I_{0}=1$. An example of this can be seen in Fig. 2 , showing $E^{\prime-1}$ as a function of $I_{0}$ measured on one of the pure tantala samples, heat-treated to $400^{\circ} \mathrm{C}$, in red circles, and the fit of Eq. (5) in blue. The green dotted lines are the one standard deviation uncertainty from the fit, assuming that the noise in the data is Gaussian.

This method requires indents at varying depth, so for each location on a sample, at least 25 indents were made, varying the maximum applied load evenly between 500 and $10,000 \mu \mathrm{N}$. At least two positions were measured on each sample, and the weighted mean of the extracted coating moduli was calculated and is given in Table 1.

For the pure tantala samples, indentations were made into coatings deposited onto both silicon and silica substrates. Finite element analysis suggests that indents into coatings on the more compliant silica substrates will have an additional substrate effect that would artificially reduce the extracted coating modulus by as much as 5\% [29]. Comparing the moduli measured on silica to those measured on

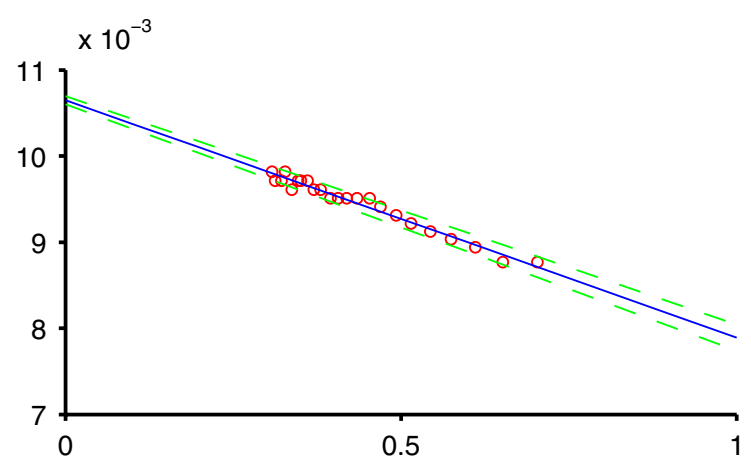

Fig. 2. Example of the fit of Eq. (5) to indentation data. Red circles are the values of $E^{\prime-1}$ from the Oliver and Pharr analysis against $I_{0}$; blue line is the fit of the Song and Pharr model to the data; green dashed lines show the one standard deviation uncertainties to the fit. These data are from one position on the pure tantala sample heat-treated to $400^{\circ} \mathrm{C}$. 
Table 1. Young's Moduli Measured for Various Titania-Doped Tantala Films ${ }^{a}$

\begin{tabular}{|c|c|c|c|c|c|c|}
\hline$\% \mathrm{Ti}$ & $\mathrm{H}-\mathrm{T}\left[{ }^{\circ} \mathrm{C}\right]$ & Substrate & Pos. Meas. & $E[\mathrm{GPa}]$ & Stat. Uncert. [GPa] & Sys. Uncert. \% \\
\hline 0 & 400 & $\mathrm{SiO}_{2}$ & 5 & 137 & 1.1 & $\pm 3+5$ \\
\hline 0 & 800 & $\mathrm{SiO}_{2}$ & 5 & 162 & 6.4 & $\pm 3+5$ \\
\hline 0 & 300 & $\mathrm{Si}$ & 2 & 160 & 14.2 & \pm 3 \\
\hline 0 & 400 & $\mathrm{Si}$ & 2 & 146 & 3.3 & \pm 3 \\
\hline 25 & 300 & $\mathrm{SiO}_{2}$ & 2 & 137 & 1.7 & $\pm 3+5$ \\
\hline 25 & 400 & $\mathrm{SiO}_{2}$ & 3 & 145 & 3.2 & $\pm 3+5$ \\
\hline 25 & 600 & $\mathrm{SiO}_{2}$ & 7 & 132 & 1.1 & $\pm 3+5$ \\
\hline 55 & $\mathrm{AD}$ & $\mathrm{SiO}_{2}$ & 3 & 145 & 4.9 & $\pm 3+5$ \\
\hline 55 & 300 & $\mathrm{SiO}_{2}$ & 3 & 158 & 2.9 & $\pm 3+5$ \\
\hline 55 & 400 & $\mathrm{SiO}_{2}$ & 3 & 142 & 1.7 & $\pm 3+5$ \\
\hline
\end{tabular}

${ }^{a}$ Included are the temperatures of heat-treatment for the samples, the number of positions measured on each sample, the Young's moduli measured, along with the statistical uncertainty from the measured indents on each sample, and the systematic uncertainty, which arises from the softer substrate and uncertainty in the Poisson ratio of the coating materials.

silicon, the moduli measured on the silica substrate samples are in fact 5\% lower. This uncertainty is included as a $+5 \%$ systematic uncertainty for all the samples on silica substrates in the table. The modulus extracted using the Song and Pharr method also depends upon the Poisson ratio of the coating material. The Poisson ratios of amorphous tantala and titaniadoped tantala are unknown; however, the Poisson ratios of similar amorphous metal oxides tend to be in the range of $0.20-0.30$. The values listed in the table are given using an assumed value for the Poisson ratio of 0.25 . Variation within the range of $0.20-0.30$ varies the resulting moduli by less than $3 \%$ in all cases.

\section{B. Thermal Bending}

Measurements of the CTE were made using silicon cantilevers of dimensions $34 \mathrm{~mm}$ long $x 5 \mathrm{~mm}$ wide and $115 \mu \mathrm{m}$ thick, which are similar to those used in cryogenic mechanical loss measurements [7,20,30,31]. The CTE of a coating deposited upon such a cantilever can be determined by measuring the variation in coating stress with sample temperature. Following the modified Stoney's formula [32,33],

$$
\sigma_{\text {coating }}=\frac{1}{6} B_{s} \frac{t_{s}^{2}}{t_{c}}\left(\frac{1}{R_{0}}-\frac{1}{R}\right)
$$

the change in the radius of curvature of a coated substrate, $R$, from that of the uncoated substrate, $R_{0}$, is related to the stress in the coating $\sigma_{\text {coating }}$, where $B_{s}$ is the biaxial modulus of the substrate, $t_{s}$ is the thickness of the substrate, and $t_{c}$ is the thickness of the coating. The biaxial modulus of a material is defined as $B=E /(1-\nu)$. Some of this stress is related to the thermal expansion mismatch, $\alpha_{c}-\alpha_{s}$, between the coating and the substrate,

$$
\sigma_{\text {coating }}=\sigma_{I}+\left(\alpha_{c}-\alpha_{s}\right) B_{c} \Delta T,
$$

where $\sigma_{I}$ is the intrinsic stress in the coating from nonthermal sources, and $\Delta T$ is the temperature difference from the last significant thermal treatment of the coating, such as deposition or heat-treatment [34]. Therefore the variation in the stress as a function of temperature yields the following relation:

$$
d \sigma_{\text {coating }} / d T=\left(\alpha_{s}-\alpha_{c}\right) B_{c} .
$$

A simple apparatus was designed in order to measure the radius of curvature of coated cantilever samples. In this apparatus, shown schematically in Fig. $\underline{3}$, a laser beam is separated into two parallel beams using a beam splitter and $45^{\circ}$ mirror, separated by a distance, $x$. These beams are reflected from the cantilever, with one spot reflecting from very near the clamped base of the cantilever, and the other spot reflecting from the tip. These two reflected beams are incident upon a screen placed a distance $L$ from the sample.

The distance between the spots on the screen, $D$, will be the sum of the original separation of the beams and the deviation caused by the curvature of the cantilever: $D=x+\delta$, where $D$ is negative if the beams cross between the sample and the screen. Therefore, if $D$ is negative or less than $x$, the beams are convergent, and the sample is concave (as drawn in Fig. 3 ); if $D$ is greater than $x$, then the beams are

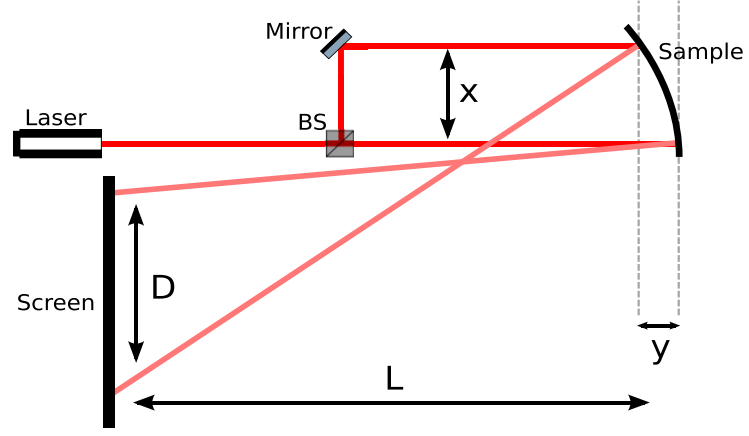

Fig. 3. Diagram of the thermal-bending experimental setup. The measurement of the spot separation, $D$, is dependent upon the displacement of the cantilever tip, $y$. This diagram is not to scale. 
divergent, and the sample is convex. If the displacement of the sample tip, $y$, is small relative to $x$, the radius of curvature of the sample can be calculated using the relation:

$$
R=2 L x / \delta .
$$

This device was able to measure the radius of curvature of the samples to an accuracy of 5\%, as tested in measurements of concave mirrors of known radius. In order to make measurements at varying temperatures, the samples were placed within an insulated copper box with a thin transparent opening to allow the passage of the laser beams. The temperature within the box was controlled using resistive heaters mounted inside the box, and the temperature was measured using a thermocouple mounted within the cantilever clamp.

Measurements of the radius of curvature were made at intervals between $25^{\circ} \mathrm{C}$ and $100^{\circ} \mathrm{C}$. The radius was converted to stress, and the stress variation with temperature was fit with a straight line to give the slope in Eq. (9). An example of the resulting plot of stress as a function of temperature is given in Fig. 4 . In this plot, the error bars are from the statistical uncertainty in the measurements as well as the systematic uncertainty in all the components of Eq. (7) except for the thickness of the substrate. Our uncertainty in the thickness of the substrate can add a systematic error of as much as $20 \%$ to the measurements of tensile stress; however, even these uncertainties are utilized in the calculation of the thermal expansion. By taking the Young's modulus measured using the nanoindention procedure discussed above, and assuming a Poisson ratio of $0.25 \pm 0.05$, the coefficient of thermal expansion (CTE) could be calculated for each sample. The results are shown in Fig. $\underline{6}$.

\section{Results}

\section{A. Young's Modulus}

Coating Young's moduli as measured on silica substrates are shown in Fig. 5 as a function of heat-treatment. Looking only at the pure tantala (red circles), the Young's modulus appears to decrease with increasing heat-treatment until the coating begins to crystallize between $600^{\circ} \mathrm{C}$ and $800^{\circ} \mathrm{C}$. Such a trend

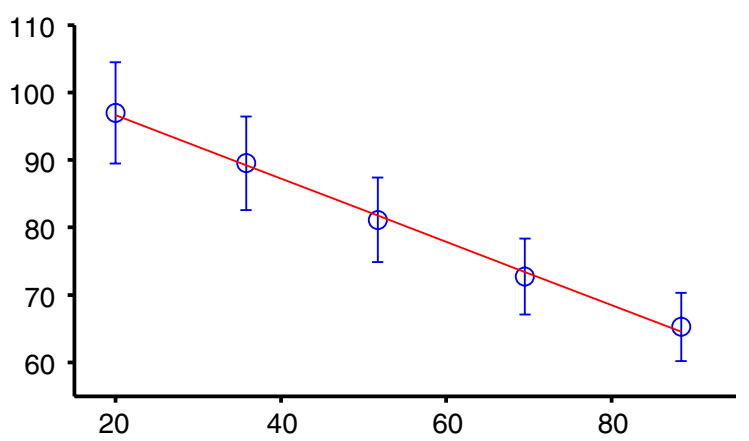

Fig. 4. Plot of tensile stress (blue circles) versus temperature with accompanying fitted line (red). The slope of this line is used to calculate the thermal expansion coefficient of the coating material.

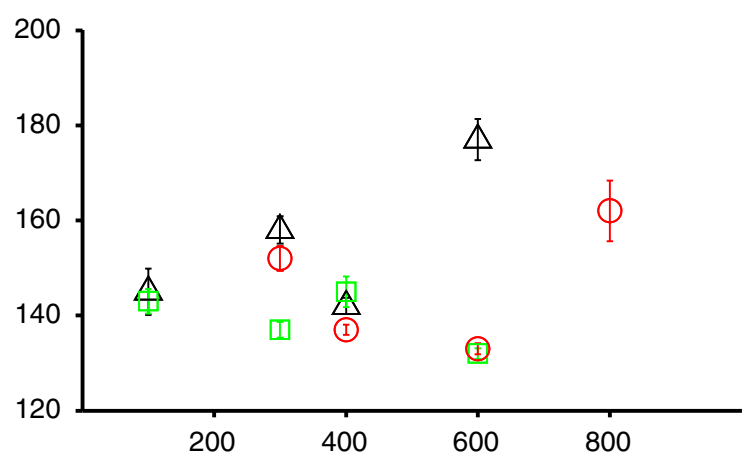

Fig. 5. Mean Young's moduli of all tantala samples measured on silica substrates, plotted for samples of different heat-treatment. The red circles are pure tantala, the green squares are $25 \%$ titaniadoped tantala, and the black triangles are 55\% titania-doped tantala. Error bars are the statistical uncertainty on the weighted mean. The sample heat-treated at $800^{\circ} \mathrm{C}$ was found to be polycrystalline; all others are amorphous.

was postulated in [35], as an indicator of increasing void space with increased heat-treatment. A similar trend is seen with the $25 \%$ titania-doped samples (green squares), with the exception of the $400^{\circ} \mathrm{C}$ sample. The opposite trend is seen with the $55 \%$ titaniadoped samples (black, upward triangles); again, with the exception of the $400^{\circ} \mathrm{C}$ sample. This is most likely due to the abundance of titania, which is known to have a low crystallization temperature and a high Young's modulus [36,37]. The two titania-doped samples heat-treated at $400^{\circ} \mathrm{C}$ were produced at the same time; they were most likely heat-treated together and may not have been fully heat-treated. This agrees with the fact that the two samples give approximately the same moduli as the as-deposited coatings. Overall, it is shown that, while the commonly measured value of $140 \mathrm{GPa}[18,38]$ is a reasonable value for the Young's modulus of tantala, it is dependent upon both the doping and heat-treatment of the coating.

\section{B. Thermal Expansion}

The coefficients of thermal expansion for both of the titania-doping concentrations are shown in Fig. $\underline{6}$. The plot suggests there is no obvious trend in the

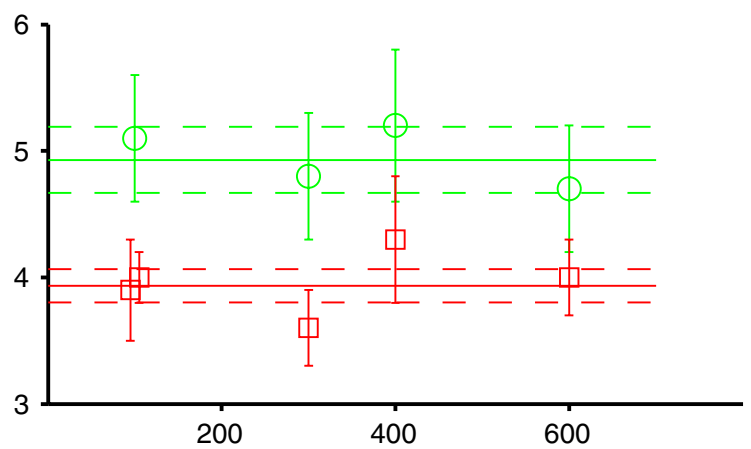

Fig. 6. Coefficient of thermal expansion measured using the thermal-bending technique for 55\% (green circles) and $25 \%$ (red squares) titania-doped tantala samples heat-treated at different temperatures. The solid lines indicate the weighted mean of each set. 
CTE of the coatings with heat-treatment temperature. Also plotted as solid lines are the weighted means of the coefficients. For the 55\% titania-doped tantala, the mean CTE is $(4.9 \pm 0.3) \times 10^{-6} \mathrm{~K}^{-1}$, and for the $25 \%$ titania-doped samples, the mean is $(3.9 \pm 0.1) \times 10^{-6} \mathrm{~K}^{-1}$. These values can be compared with the reported CTE of pure tantala coatings deposited by dual ion-beam sputtering in [18], which gives a value of $4.4 \times 10^{-6} \mathrm{~K}^{-1}$. Both of these values are higher than that used for pure tantala within the LIGO community; however, they are probably affected by the presence of the titania doping. Unfortunately, we were unable to measure any samples of pure tantala.

\section{Conclusion}

We have made direct measurements of the CTE and Young's modulus of pure and titania-doped tantala heat-treated at various temperatures. Our results indicate that the Young's modulus of IBS tantala films is affected by post-deposition heat-treatment and titania doping, and that the CTE is not affected by heattreatment but is altered by titania doping. We have also made direct measurements of coating materials, which are similar to those used in second-generation interferometric gravitational wave detectors, confirming previous estimates, and allowing for greater confidence in the prediction of thermal noise levels within these detectors.

The authors would like to acknowledge the contributions of the Oyen Group from the Cambridge University Department of Engineering, especially Oliver Hudson, Tamaryn Shean, and Daniel Strange for their aid in obtaining the nanoindentation data. IWM holds a Royal Society University Research Fellowship, and SR is a Royal Society-Wolfson Research Merit Award holder. The authors would like to thank the UK Science and Technology Facilities Council, the University of Glasgow, the Scottish Universities Physics Alliance, and the Scottish Founding Council for financial support through RCUK grants ST/J000361/1 and CG ST/L000946/1. We also wish to thank our colleagues in the GEO600 and LIGO Scientific Collaboration for their interest in this work. The LIGO Observatories were constructed by the California Institute of Technology and Massachusetts Institute of Technology with funding from the National Science Foundation under cooperative agreement PHY9210038. The LIGO Laboratory operates under cooperative agreement PHY-0107417. This paper has been assigned LIGO Document Number LIGOP1300107.

\section{References}

1. G. M. Harry (for the LIGO Scientific Collaboration), "Advanced LIGO: the next generation of gravitational wave detectors," Class. Quantum Grav. 27, 084006 (2010).

2. The Virgo Collaboration, "Advanced virgo baseline design," https://tds.ego-gw.it/itf/tds/file.php?callFile=VIR-0027A-09.pdf (2009). Note VIR-027A-09.

3. A. E. Villar, E. D. Black, R. DeSalvo, K. G. Libbrecht, C. Michel, N. Morgado, L. Pinard, I. M. Pinto, V. Pierro, V. Galdi,
M. Principe, and I. Taurasi, "Measurement of thermal noise in multilayer coatings with optimized layer thickness," Phys. Rev. D 81, 122001 (2010).

4. M. Evans, S. Ballmer, M. Fejer, P. Fritschel, G. Harry, and G. Ogin, "Thermo-optic noise in coated mirrors for highprecision optical measurements," Phys. Rev. D 78, 102003 (2008).

5. G. M. Harry, A. M. Gretarsson, P. R. Saulson, S. E. Kittelberger, S. D. Penn, W. J. Startin, S. Rowan, M. M. Fejer, D. R. M. Crooks, G. Cagnoli, J. Hough, and N. Nakagawa, "Thermal noise in interferometric gravitational wave detectors due to dielectric optical coatings," Class. Quantum Grav. 19, 897-917 (2002).

6. S. D. Penn, P. H. Sneddon, H. Armandula, J. C. Betzwieser, G. Cagnoli, J. Camp, D. R. M. Crooks, M. M. Fejer, A. M. Gretarsson, G. M. Harry, J. Hough, S. E. Kittelberger, M. J. Mortonson, R. Route, S. Rowan, and C. C. Vassiliou, "Mechanical loss in tantala/silica dielectric mirror coatings," Class. Quantum Grav. 20, 2917-2928 (2003).

7. I. W. Martin, E. Chalkley, R. Nawrodt, H. Armandula, R. Bassiri, C. Comtet, M. M. Fejer, A. Gretarsson, G. Harry, D. Heinert, J. Hough, I. MacLaren, C. Michel, J.-L. Montorio, N. Morgado, S. Penn, S. Reid, R. Route, S. Rowan, C. Schwarz, P. Seidel, W. Vodel, and A. L. Woodcraft, "Comparison of the temperature dependence of the mechanical dissipation in thin films of $\mathrm{Ta}_{2} \mathrm{O}_{5}$ and $\mathrm{Ta}_{2} \mathrm{O}_{5}$ doped with $\mathrm{TiO}_{2}$," Class. Quantum Grav. 26, 155012 (2009).

8. M. N. Inci, "Simultaneous measurements of the thermal optical and linear thermal expansion coefficients of a thin film etalon from the reflection spectra of a super-luminescent diode," J. Phys. D 37, 3151-3154 (2004).

9. C.-L. Tien, C.-C. Lee, K.-P. Chuang, and C.-C. Jaing, "Simultaneous determination of the thermal expansion coefficient and the elastic modulus of ta205 thin film using phase shifting interferometry," J. Mod. Opt. 47, 1681-1691 (2000).

10. V. B. Braginsky and A. A. Samoylenko, "Measurements of the mirror optical coating properties," Phys. Lett. A 315, 175-177 (2003).

11. M. Fejer, S. Rowan, G. Cagnoli, D. Crooks, A. Gretarsson, G. Harry, J. Hough, S. Penn, P. Sneddon, and S. Vyatchanin, "Thermoelastic dissipation in inhomogeneous media: loss measurements and displacement noise in coated test masses for interferometric gravitational wave detectors," Phys. Rev. D 70, 082003 (2004).

12. M. A. Scobey and P. D. Stupik, "Stable ultranarrow bandpass filters," Proc. SPIE 2262, 37-46 (1994).

13. A. Chu, H. Lin, and W. Cheng, "Temperature dependence of refractive index of $\mathrm{Ta}_{2} \mathrm{O}_{5}$ Dielectric Films," J. Electron. Mater. 26, 889-892 (1997).

14. P. Martin, A. Bendavid, M. Swain, R. Netterfield, T. Kinder, W. Sainty, and D. Drage, "Mechanical and optical properties of thin films of tantalum oxide deposited by ion-assisted deposition," in Materials Research Society Symposium Proceedings (1993), Vol. 306, p. 583.

15. D. R. M. Crooks, G. Cagnoli, M. Fejer, A. Gretarsson, G. Harry, J. Hough, N. Nakagawa, S. Penn, R. Route, S. Rowan, and P. H. Sneddon, "Experimental measurements of coating mechanical loss factors," Class. Quantum Grav. 21, S1059-S1065 (2004).

16. I. Martin, H. Armandula, C. Comtet, M. M. Fejer, A. Gretarsson, G. Harry, J. Hough, J. M. Mackowski, I. MacLaren, C. Michel, J.-L. Montorio, N. Morgado, R. Nawrodt, S. Penn, S. Reid, A. Remillieux, R. Route, S. Rowan, C. Schwarz, P. Seidel, W. Vodel, and A. Zimmer, "Measurements of a low-temperature mechanical dissipation peak in a single layer of $\mathrm{Ta}_{2} \mathrm{O}_{5}$ doped with $\mathrm{TiO}_{2}$," Class. Quantum Grav. 25, 055005 (2008).

17. M. Gross, "CSIRO Report 1, Ion beam deposited coatings," LIGO document LIGO-T040236-00-D (2004), https://dcc.ligo .org/public/0027/T040236/000/T040236-00.pdf.

18. E. Çetinörgü, B. Baloukas, O. Zabeida, J. E. KlembergSapieha, and L. Martinu, "Mechanical and thermoelastic characteristics of optical thin films deposited by dual ion beam sputtering," Appl. Opt. 48, 4536-4544 (2009). 
19. G. M. Harry, M. R. Abernathy, A. E. Becerra-Toledo, H. Armandula, E. Black, K. Dooley, M. Eichenfield, C. Nwabugwu, A. Villar, D. R. M. Crooks, G. Cagnoli, J. Hough, C. R. How, I. MacLaren, P. Murray, S. Reid, S. Rowan, P. H. Sneddon, M. M. Fejer, R. Route, S. D. Penn, P. Ganau, J. Mackowski, C. Michel, L. Pinard, and A. Remillieux, "Titaniadoped tantala/silica coatings for gravitational-wave detection," Class. Quantum Grav. 24, 405-415 (2007).

20. I. W. Martin, R. Bassiri, R. Nawrodt, M. M. Fejer, A. Gretarsson, E. Gustafson, G. Harry, J. Hough, I. MacLaren, S. Penn, S. Reid, R. Route, S. Rowan, C. Schwarz, P. Seidel, J. Scott, and A. L. Woodcraft, "Effect of heat treatment on mechanical dissipation in $\mathrm{Ta}_{2} \mathrm{O}_{5}$ coatings," Class. Quantum Grav. 27, 225020 (2010).

21. J. Menčík, D. Munz, E. Quandt, E. Weppelmann, and M. Swain, "Determination of elastic modulus of thin layers using nanoindentation," J. Mater. Res. 12, 2475-2484 (1997).

22. W. C. Oliver and G. M. Pharr, "Measurement of hardness and elastic modulus by instrumented indentation: advances in understanding and refinements to methodology," J. Mater. Res. 19, 3-20 (2004).

23. W. Oliver and G. Pharr, "An improved technique for determining hardness and elastic modulus using load and displacement sensing indentation experiments," J. Mater. Res. 7, 1564-1583 (1992).

24. R. Saha and W. Nix, "Effects of the substrate on the determination of thin film mechanical properties by nanoindentation," Acta Materialia 50, 23-38 (2002).

25. A. Fischer-Cripps, "Critical review of analysis and interpretation of nanoindentation test data," Surf. Coat. Technol. 200, 4153-4165 (2006).

26. H. Song, "Selected mechanical problems in load and depth sensing indentation testing," Ph.D. thesis (Rice University, 1999).

27. A. Rar, H. Song, and G. M. Pharr, "Assessment of new relation for the elastic compliance of a film-substrate system," in MRS Online Proceedings Library (2001), Vol. 695, p. L10.10.1.
28. H. Gao, C.-H. Chiu, and J. Lee, "Elastic contact versus indentation modeling of multi-layered materials," Int. J. Solids Struct. 29, 2471-2492 (1992).

29. J. Hay and B. Crawford, "Measuring substrate-independent modulus of thin films," J. Mater. Res. 26, 727-738 (2011).

30. M. R. Abernathy, S. Reid, E. Chalkley, R. Bassiri, I. W. Martin, K. Evans, M. M. Fejer, A. Gretarsson, G. M. Harry, J. Hough, I. MacLaren, A. Markosyan, P. Murray, R. Nawrodt, S. Penn, R. Route, S. Rowan, and P. Seidel, "Cryogenic mechanical loss measurements of heat-treated hafnium dioxide," Class. Quantum Grav. 28, 195017 (2011).

31. S. Reid, G. Cagnoli, D. Crooks, J. Hough, P. Murray, S. Rowan, M. Fejer, R. Route, and S. Zappe, "Mechanical dissipation in silicon flexures," Phys. Lett. A 351, 205-211 (2006).

32. G. G. Stoney, "The tension of metallic films deposited by electrolysis," Proc. Roy. Soc. London A 82, 172-175 (1909).

33. R. W. Hoffman, "The mechanical properties of thin condensed films," in Physics of Thin Films (Academic, 1966), Vol. 3.

34. M. F. Doerner and W. D. Nix, "Stresses and deformation processes in thin films on substrates," Critical Reviews in Solid State and Materials Sciences 14, 225-268 (1988).

35. J. T. Brown, "Center wavelength shift dependence on substrate coefficient of thermal expansion for optical thin-film interference filters deposited by ion-beam sputtering," Appl. Opt. 43, 4506-4511 (2004).

36. Y. Gaillard, V. J. Rico, E. Jimenez-Pique, and A. R. GonzálezElipe, "Nanoindentation of $\mathrm{TiO}_{2}$ thin films with different microstructures," J. Phys. D 42, 145305 (2009).

37. K. Kurosaki, D. Setoyama, J. Matsunaga, and S. Yamanaka, "Nanoindentation tests for $\mathrm{TiO}_{2}, \mathrm{MgO}$, and YSZ single crystals," J. Alloys Compd. 386, 261-264 (2005).

38. J. E. Klemberg-Sapieha, J. Oberste-Berghaus, L. Martinu, R. Blacker, I. Stevenson, G. Sadkhin, D. Morton, S. McEldowney, R. Klinger, P. J. Martin, N. Court, S. Dligatch, M. Gross, and R. P. Netterfield, "Mechanical characteristics of optical coatings prepared by various techniques: a comparative study," Appl. Opt. 43, 2670-2679 (2004). 